\title{
Application of NGS for studying the antibiotic resistant microbiome in Wrocław tap water
}

\author{
Agata Siedlecka ${ }^{1, *}$, and Katarzyna Piekarska ${ }^{1}$ \\ ${ }^{1}$ Wrocław University of Science and Technology, Faculty of Environmental Engineering, \\ Wyb. Wyspiańskiego 27, 50-370 Wrocław, Poland
}

\begin{abstract}
The NGS method was used to investigate the biodiversity of ARB dwelling in tap water, able to form biofilm on bullion media supplemented with antibiotics: $\beta$-lactam, fluoroquinolone, $3^{\text {rd }}$ generation cephalosporin and tetracycline. Biofilms formed by cold- and hot-tap water microorganisms were also examined. The presence of 10 ARGs in plasmid DNA of cultivated biomass was tested using a standard PCR. The analyses revealed that the most predominant families were Sphingomonadaceae, Paenibacillaceae, Enterobacteriaceae, Bacillaceae and Sphingomonadaceae in FQ, C3G, T, C-W and H-W samples, respectively (explanations of abbreviations in the text). The ARGs did not correlate with resistance phenotypes.
\end{abstract}

\section{Introduction}

Antibiotic resistance of bacteria became a worldwide problem. The resistance of environmental strains is regarded as a threat, as these bacteria could act as the vectors of total resistance determinants [1]. Therefore, attempts are made to evaluate the total (intrinsic and acquired) resistance in environmental strains. As tap water is a direct source of drinking water, the occurrence of resistomes, i.e. antibiotic resistant bacteria (ARB), and antibiotic resistance genes (ARGs) in this medium should be regarded with particular care.

Many studies of antibiotic resistance phenomenon in tap water are available, but they mainly concern enumerating ARB and ARGs or investigate their (relative) abundances, as for example [2]. In this study, a different approach was adopted. The cultivation of biofilms formed by ARB allow detecting the part of bacteria, which could act as opportunistic pathogens, outside of just being resistant. The aerobic, heterotrophic, mesophilic ARB, which proliferate more advantageous than other bacteria could, in some circumstances, be regarded as health threatening [3]. The presence of these bacteria in tap water was evaluated in simple experiments of biofilm cultivation on bullion media supplemented with antibiotics.

The biodiversity of mixed bacterial cultures was determined by the next generation sequencing (NGS) approach. The method allows identifying (theoretically) all of the microorganisms, including non-cultivable species, present in a given sample on

\footnotetext{
*Corresponding author: agata.siedlecka@pwr.edu.pl
} 
the basis of DNA sequence. As the aim of this study was to establish relative abundances of bacteria in cultures, the targeted $16 \mathrm{~S}$ rRNA metagenomic approach was applied. This involves the PCR amplification of V3/V4 variable regions of $16 \mathrm{~S}$ rRNA gene during a library preparation step. The NGS platform of choice was MiSeq from Illumina. The Illumina technology uses bridge amplification for cluster generation by isothermal amplification and sequencing using a synthesis approach. The prepared library is loaded onto the flow cell covered with oligos, which are complementary to adapters (added to V3/V4 amplicons during the library prep step). During the sequencing step, fluorescently labelled (and acting as reversible terminators) dNTPs are incorporated into the growing DNA chain. The image is taken, and the colour and intensity of fluorescent signal is recorded. The most time-consuming stage of the process is the imaging of the flow cell [4]. Many samples may be sequenced and analysed in the same run due to implementation of indices (short oligos characteristic for a given sample) and subsequent demultiplexing [5].

The obtained DNA sequences are analysed by BaseSpace (Illumina) software. After quality filtering, the sequences reads are alignment to the GreenGenes database for the identification purpose. The main disadvantage of this database is that it was last updated in May 2013 [6].

Despite the possibility to identify microorganisms and evaluate their relative abundances simultaneously in many samples in a rapid way, the NGS technology is not free from setbacks. Firstly, bioinformatic analyses of raw data require users' experience. Indeed, the interpretation of the same data with different bioinformatic databases as NCBI, RDP, SILVA or GreenGenes or with different parameters may lead to different results [6]. Secondly, library preparation and sequencing steps could introduce errors [5]. Finally, the sequencing technologies, although constantly improved in terms of data output and accuracy, are not sufficiently reliable themselves. For example, there is an evidence, that the application of different NGS platforms could yield different results, even if the same DNA was investigated [7]. The sequences might be identified at kingdom, phylum, class, order, family, genus, or species level, but in metagenomic NGS studies, the identification at the genus level is considered to be more reliable, than at the species level [6].

The ARGs selected in this study correspond to resistance to chosen antibiotics. The investigated antibiotics and genes conferring resistance are summarised in Table $1[8,9]$.

Table 1. Antibiotics and ARGs chosen in this study.

\begin{tabular}{|c|c|c|c|}
\hline $\begin{array}{c}\text { group of } \\
\text { antibiotics }\end{array}$ & antibiotic & ARGs & mechanism of resistance \\
\hline$\beta$-lactams & amoxicillin & - & - \\
\hline \multirow{2}{*}{ FQ } & \multirow{2}{*}{ ciprofloxacin } & $a a c\left(6^{\prime}\right)-I b-c r$ & $\begin{array}{l}\text { aminoglycoside acetyltransferase that acetylate } \\
\text { fluoroquinolones with a piperazinyl substituent }\end{array}$ \\
\hline & & qnrss & $\begin{array}{l}\text { proteins that protect DNA gyrase and } \\
\text { topoisomerase IV from quinolones }\end{array}$ \\
\hline $\mathrm{C} 3 \mathrm{G}$ & ceftazidime & $\begin{array}{l}\text { bla }_{\text {CTX-M }}, \text { bla } \\
\text { blaOXA } \\
\text {, blaSHV }\end{array}$ & $\beta$-lactamases \\
\hline $\mathrm{T}$ & tetracycline & $\begin{array}{c}\text { tet }_{A}, \text { tetK }_{K}, \text { tet }_{L} \\
\text { tets }\end{array}$ & $\begin{array}{l}\text { a ribosomal protection mechanism and/or } \\
\text { efflux mechanism }\end{array}$ \\
\hline
\end{tabular}




\section{Materials and methods}

\subsection{Sample collection}

All drinking water samples were collected from the tap located in the laboratory at Faculty of Environmental Engineering, Wrocław University of Science and Technology in November 2017. Before sampling, the tap was disinfected, and water was flushed for $20 \mathrm{~s}$ with the maximum speed and 20 minutes with the medium speed. 4 samples of $5 \mathrm{~L}$ volume each were collected in sterile glass bottles and processed further immediately afterwards. Additionally, one sample of cold tap water and one sample of hot tap water, $1 \mathrm{~L}$ each, were collected in the same conditions.

\subsection{Cultivation of bacteria}

Biomass from all samples of water was concentrated under sterile conditions through cellulose nitrate membrane filter of $0.2 \mu \mathrm{m}$ pore diameters (Whatman). The membranes were placed in conical flasks containing $30 \mathrm{ml}$ of sterile bullions, consisting of $1.7 \mathrm{~g}$ yeast extract, $0.4 \mathrm{~g}$ meat extract, $4.0 \mathrm{~g}$ peptone, $5.4 \mathrm{~g}$ peptone $\mathrm{K}, 3.5 \mathrm{~g} \mathrm{NaCl}$ in $1 \mathrm{~L}$ of distilled water (BTL). For the cultivation of ARB biofilms, bullions were supplemented with amoxicillin $(8 \mathrm{mg} / \mathrm{L})$, ciprofloxacin $(1 \mathrm{mg} / \mathrm{L})$, ceftazidime $(8 \mathrm{mg} / \mathrm{L})$, tetracycline $(16 \mathrm{mg} / \mathrm{L})$ for the detection of bacteria resistant to $\beta$-lactams, fluoroquinolones (FQ), $3^{\text {rd }}$ generation cephalosporins $(\mathrm{C} 3 \mathrm{G})$ and tetracyclines $(\mathrm{T})$, respectively. The drug concentrations were established in accordance to EUCAST, CLSI or the literature guidelines [8]. To determine the biodiversity of bacteria which can form biofilm in tap water, additional $1 \mathrm{~L}$ samples of cold $(\mathrm{C}-\mathrm{W})$ and hot water $(\mathrm{H}-\mathrm{W})$ were processed as mentioned above and the membranes were placed in conical flasks containing $30 \mathrm{ml}$ of bullion (BTL) without antibiotic supplementation. Flasks with membranes were subjected to ultrasounds for $1 \mathrm{~min}$ and to shaking to enable the detachment and distribution of cells from membrane through the medium. All flasks were incubated with membranes at $37^{\circ} \mathrm{C}$ for $72 \mathrm{~h}$.

\subsection{DNA extraction}

The biomass of every sample in which the growth of microorganisms was observed was centrifuged and the pellet was subjected to DNA extraction. Genomic and plasmid DNA was extracted with Genomic Mini and Plasmid Mini isolation kits (A\&A Biotechnology), respectively, to determine the biodiversity of biofilm and the presence of ARGs. The concentration and quality of extracted DNA were determined by NanoDrop 1000 (Thermo Fisher Scientific).

\subsection{Determination of biodiversity and ARGs}

To determine the biodiversity of obtained biofilms, genomic DNA was subjected to Illumina Next Generation Sequencing in MiSeq platform (Genomed, Poland) using the metagenomic targeted $16 \mathrm{~S}$ rRNA gene approach. The V3/V4 variable region of 16S rRNA gene was amplified during the library preparation procedure. To determine the relative abundance of identified genera/species, the basic bioinformatic analysis was applied, involving tools provided by Illumina (BaseSpace), using GreenGenes database.

For ARGs determination, standard PCRs were applied. PCR mixtures consisted of $\mathrm{ddH}_{2} \mathrm{O}$ and 2xMasterMix (A\&A Biotechnology), specific primers of concentrations ranging 
from $10 \mu \mathrm{M}$ to $100 \mu \mathrm{M}$ and $2 \mu \mathrm{l}$ of plasmid DNA. 10 ARGs were investigated in this study, as presented in Table 1 (for primers sequences and PCR conditions, please see [8]).

In each case, negative controls (without DNA) were applied. All PCR products were determined by gel electrophoresis $(15 \mathrm{~min}$ at $130 \mathrm{~V}$ and then $60 \mathrm{~min}$ at $80 \mathrm{~V}$ or $90 \mathrm{~min}$ at $80 \mathrm{~V})$ in $1 \%(\mathrm{w} / \mathrm{v})$ agarose gel (Merck) in $1 \mathrm{xTBE}$ buffer and visualized with ethidium bromide staining [8].

\section{Results and discussion}

\subsection{Cultivation of ARB}

After the incubation, no growth of microorganisms was observed in the sample supplemented with amoxicillin ( $\beta$-lactam), therefore the sample was excluded in further investigations. In other samples, the lowest growth was observed in tetracycline supplemented bullion, the stronger - in bullions without antibiotic supplementation. The observed differences indicate the diverse growth rates of ARB and overall bacteria isolated from tap water in rich medium at temperature suitable for mesophilic strains. The temperature and time of incubation applied in the study were established to enable the cultivation of opportunistic pathogens from water samples and to determine which bacteria would proliferate and dominate the other species.

\subsection{Biodiversity of cultivated biofilms and their ARGs}

The results of biodiversity investigations of all tested samples are presented below.

Fig. 1 and 2 demonstrate the results of classification to genus and species, respectively.

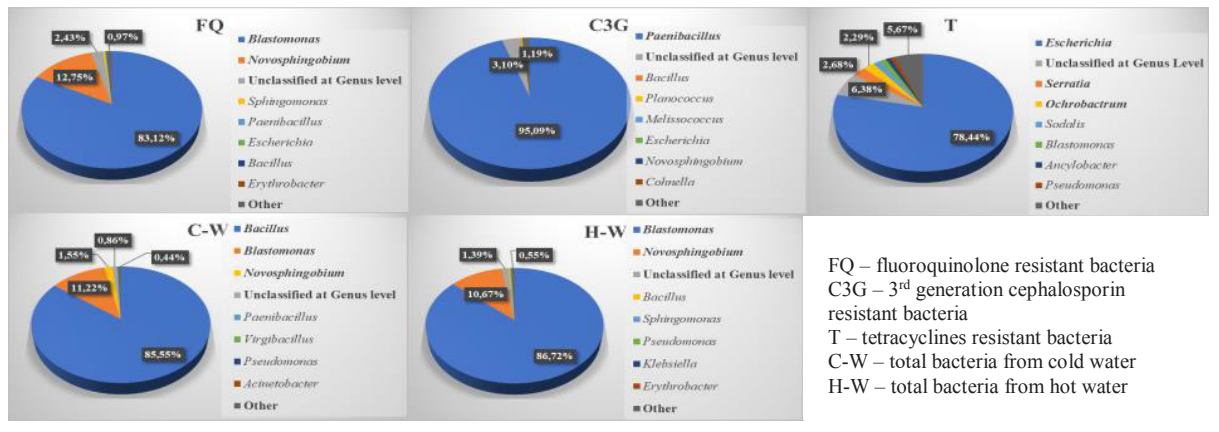

Fig. 1. Results of classifications at the genus level.

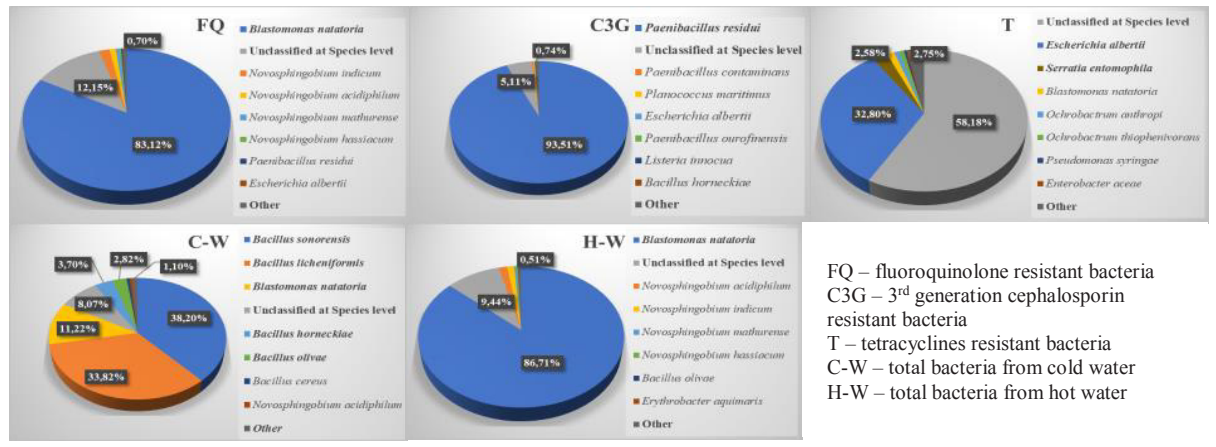

Fig. 2. Results of classifications at the species level. 
It is worth noting that the results of classification could vary significantly, when other databases (or parameters) [6] or sequencing platforms [7] were applied. As demonstrated in Fig. 1 and 2, the obtained results depend on the level of classification. Therefore, the NGS data, although regarded as the breakthrough in environmental studies, need to be analysed carefully. In general, lower biodiversity was expected in ARB biofilm samples. However, the high numbers of classified categories might be the effect of the sensitivity of the method - it is possible that residual DNA concentrated on the membrane filters and transferred to the samples has affected the results.

The presence of selected ARGs analysed in this study is demonstrated in Table 2.

Table 2. The presence of ARGs in the tested samples.

\begin{tabular}{|l|c|c|c|c|c|}
\hline \multicolumn{1}{|c|}{ sample } & FQ & C3G & T & C-W & H-W \\
\hline${\text { aac }\left(6^{\prime}\right)-I b-c r}^{\prime}$ & - & - & - & - & - \\
\hline qnr $_{S}$ & + & - & - & - & - \\
\hline bla $_{C T X-M}$ & - & - & - & - & - \\
\hline bla $_{T E M}$ & - & - & + & + & + \\
\hline bla $_{S H}$ & - & - & - & - & - \\
\hline bla $_{O X A}$ & + & + & - & - & - \\
\hline tet $_{A}$ & - & - & - & - & - \\
\hline tet $_{K}$ & - & - & - & - & - \\
\hline tet $_{L}$ & - & - & - & - & - \\
\hline tet & - & + & - & - & - \\
\hline
\end{tabular}

\subsubsection{Biodiversity of fluoroquinolone resistant bacteria}

From 144333 total reads, 128221 (88.8\%) passed quality filtering. Among them, 97.57\% were classified at the genus level (291 categories identified) and $87.85 \%$ were classified at the species level (266 categories identified).

The most prevalent were Blastomonas and Novosphingobium, which belong to the Sphingomonadaceae family. These bacteria are ubiquitous in natural and anthropogenic environments and are often isolated from tap water [10]. In the study of Vaz-Moreira et al., $25.6 \%$ of the Sphingomonadaceae isolates revealed resistance to cirpofolxacin. Particularly, from 12 Blastomonas natatoria and 12 Novosphingobium spp. isolates, 7 (58.3\%) and $1(8.3 \%)$ revealed resistance to this antibiotic, respectively. Although the resistance phenotypes were determined by using the ATB PSE5 panel (dedicated to Pseudomonas bacteria), the applied ciprofloxacin concentration was the same as in this study (i.e. $1 \mathrm{mg} / \mathrm{L}$ ). The authors suggested that the incubation condition (i.e. selective or nonselective medium and temperature) could influence the resistance patterns of the isolates. It is worth noting that colistin resistance was supposed to be intrinsic in the Sphingomonadaceae family [10]. Moreover, in Blastomonas spp., intrinsic aminoglycosides resistance was observed [11]. Acquired resistance phenotypes of the family comprised fluoroquinolones, cephalosporins and sulfonamides. In the study of hospital tap water, the most represented genus was Blastomonas spp., but Novosphingobium spp. were rare and never isolated from household taps in mentioned studies $[10,11]$. The prevalence of antibiotic resistance to ciprofloxacin was significantly higher among Sphingomonadaceae isolates from household tap than hospital taps [10, 11]. Therefore, the presence of ciprofloxacin resistant Novosphingobium spp. revealed in this study is unique. However, in the study designed to evaluate the fate and influence of ciprofloxacin chlorination products on drinking water microorganisms' consortia, the Novosphingobium spp. were detected in samples of water with these agents. The authors suggest that this genus could benefit from biotransformation and biomineralization of some ciprofloxacin chlorination products. Moreover, the increase of $q n r_{S}$ gene relative abundance 
correlated with the increase of Novosphingobium spp. abundance in the tested samples [12]. These findings could explain the presence of $q n r_{S}$ gene in plasmid DNA extracted from FQ resistant biofilm revealed in this study.

\subsubsection{Biodiversity of $3^{\text {rd }}$ generation cephalosporin resistant bacteria}

From 107922 total reads, 95802 (88.8\%) passed quality filtering. Among them, $96.90 \%$ were classified at the genus level (268 categories identified) and $94.89 \%$ were classified at the species level (264 categories identified).

Most of the reads were classified as Paenibacilli. Paenibacilli are Gram-variable endospore-forming rods that may be isolated from various environments [13, 14]. Paenibacillus residui was first isolated from urban waste compost and characterized by Vaz-Moreira et al. as ciprofloxacin resistant, but susceptible to cephalothin, ceftazidime, colistin sulfate and streptomycin [13]. Nevertheless, the emergence of $P$. residui as a clinical isolate in a cancer patient during breast implant infection became a fact. This discovery questions the saprophytic characteristic of the strain. However, the clinical isolates of $P$. residui were susceptible to ciprofloxacin (at concentration of $0.12 \mathrm{mg} / \mathrm{L}$, what is significantly lower than in this study, i.e. $1 \mathrm{mg} / \mathrm{L}$ ) and resistant only to ceftazidime (at concentration $>256 \mathrm{mg} / \mathrm{L}$ ). Nevertheless, the authors suggest that the species might be unidentified due to the lack of its profiles in Vitek platforms (bioMerieux), often used in medicine microbiology [14]. These findings underline the health risk associated with ARB, even these considered as environmental saprophytic strains. In other study, Paenibacilli were resistant mostly to ampicillin [15].

\subsubsection{Biodiversity of tetracycline resistant bacteria}

From 145946 total reads, 130575 (89.5\%) passed quality filtering. Among them, 93.62\% were classified at the genus level (305 categories identified) and only $41.81 \%$ were classified at the species level (438 categories identified).

In tetracycline resistant biofilm, the most predominant species was Escherichia albertii. However, many reads remained unclassified at the species level. The whole genome of E. albertii was recently sequenced. This strain is recognized as an emerging pathogen, often isolated from patients with diarrhoea [16]. According to Ooka et al., the clinical significance of $E$. albertii is not fully elucidated due to the difficulties of discriminating the strain from other Enterobacteriaceae spp. using systems based on biochemical properties [17]. Moreover, the strains could possess virulence factors characteristic for enteropathogenic and enterohemorrhagic E. coli [16, 17]. All E. albertii isolates were resistant to tetracycline in the study of Perez et al. [18] and $13 \%$ of them were tetracycline resistant in the study of Ingle et al. [19].

\subsubsection{Biodiversity of bacteria forming biofilm in cold- and hot-tap water}

In the case of cold-tap water, from 145084 total reads, $131225(90.4 \%)$ passed quality filtering. Among them, 99.14\% were classified at the genus level (134 categories identified) and $91.93 \%$ were classified at the species level (185 categories identified).

Almost all total reads classified as Blastomonas were further classified as Blastomonas natatoria. Most of Bacillus reads were classified as Bacillus sonorensis and Bacillus licheniformis. These findings are in contrast with the results of Grabińska-Łoniewska et al. [20] but are consistent with the previous study of Wroclaw tap water (data not published). Furthermore, Firmicutes only accounted for around $2 \%$ of phyla classification in treatment plant outlet and around $1.1 \%$ in a drinking water distribution network in The Netherlands, 
determined using a pyrosequencing analysis of 16S rRNA gene [21]. Nevertheless, in a huge study of the diversity of microorganisms in drinking water systems of 17 cities in United States, the Bacillus spp. were observed to occur in less than $10 \%$ of chlorinated systems but at least in $75 \%$ of chloraminated systems, suggesting the possible influence of disinfectant on their prevalence in water distribution systems [22]. Similar observations were reported in the literature [23]. However, Proteobacteria are among the most abundant phyla of tap water microbiome in many studies [21-24].

In the case of hot-water, from 151204 total reads, 134204 (88.8\%) passed quality filtering. Among them, 98.61\% were classified at the genus level (155 categories identified) and $90.56 \%$ were classified at the species level (192 categories identified).

Interestingly, the abundances of Blastomonas and Novosphingobium genera are similar in biofilms formed by fluoroquinolone resistant bacteria from cold water and all bacteria from hot water (see pie charts in Fig. 1). Unfortunately, little is known about the biodiversity and microbial ecology of hot-tap water samples. Usually, these types of samples are investigated in terms of the presence of Legionella microbiome [25, 26]. Nevertheless, Blastomonas and Novosphingobium were not detected in warm water biofilm samples collected from shower hoses [27].

\subsubsection{The presence of ARGs in biofilm samples}

There is no correlation between resistance phenotypes and ARGs of investigated ARB biofilms. FQ and $\mathrm{C} 3 \mathrm{G}$ resistant bacteria possess only one of the tested genes conferring resistance to these drugs ( $q n r_{S}$ and bla $a_{O X A}$, respectively), while $\mathrm{T}$ resistant bacteria did not reveal any of the investigated tet genes. The presence of other types of genes is not surprising, as the bacteria present in the sample might exhibit other resistance phenotypes, as well. The same observation concerns the bacteria not subjected to drugs (i.e. biofilms from cold- and hot-water). Investigated bacteria could possess other ARGs conferring resistance phenotypes, not included in the study. In addition, although the biodiversity (in terms of the most prevalent genera) of samples FQ and H-W are similar, the other ARGs were detected in their plasmid DNA. All of the tested ARGs are plasmid encoded [8].

\section{Conclusions}

The genera and species detected in this study belong mainly to the typical, environmental strains, often found in tap water samples. However, a few of them (e.g. E. albertii) are associated with human infections and their presence in drinking water samples could be regarded as a threat. Moreover, the environmental strains might be opportunistic pathogens, mainly in immunocompromised patients. ARB dwelling in tap water are regarded as resistance vectors. The investigations of ARGs could indicate the mechanisms and possible ways of transfer of antibiotic resistance, but do not provide the complete explanation of the phenomenon. The NGS method requires the improvements of its accuracy, to make the results more reliable, but it might be a useful tool in environmental studies, when a lot of data are needed. The main advantage of NGS technology is the possibility to detect noncultivable microorganisms. It may completely replace the traditional, tedious cultivation methods.

The work was realized within the allocation No. 0402/0138/17 awarded for Faculty of Environmental Engineering Wroclaw University of Science and Technology by Ministry of Science and Higher Education in years 2017-2018. 


\section{References}

1. J.L. Martinez, Science 321, 365-67 (2008)

2. Ch. Xi, Y. Zhang, C.F. Marrs, W. Ye, C. Simon, B. Foxman, J. Nriagu, Appl. Environ. Microbiol. 75, 17, 5714-5718 (2009)

3. L. Balcázar, J.Subirats, C.M. Borrego, Front. Microbiol. 6, 1216, 1-10 (2015)

4. H.P.J. Buermans, J.T. den Dunnen, Biochim. Biophys. Acta. 1842, 1932-1941 (2014)

5. E.L. van Dijk, H. Auger, Y. Jaszczyszyn, C. Thermes, Trends Genet. 30, 9, 418-426 (2014)

6. M. Balvociute, D.H. Huson, BMC Genomics 18, 3-8 (2017)

7. A. Hahn, A. Sanyal, G.F. Perez, A.M. Colberg-Poley, J. Campos, M.C. Rose, M. Pérez-Losada, J. Microbiol. Methods 130, 95-99 (2016)

8. A. Osińska, M. Harnisz, E. Korzeniewska, Environ. Sci. Pollut. Res. 23, 11, 10818-10831 (2016)

9. L.K. Ng, I. Martin, M. Alfa, M. Mulvey, Mol. Cell Probes. 15, 4, 209-215 (2001)

10. I. Vaz-Moreira, O.C. Nunes, C.M. Manaia, Appl. Environ. Microbiol. 77, 16, 5697-5706 (2011)

11. C. Narciso-da-Rocha, I. Vaz-Moreira, C.M. Manaia, Sci. Total Environ. 466-467, 127-135 (2014)

12. H. Wang, Ch. Hu, L. Liu, X. Xing, J. Hazard. Mater. 339, 174-181 (2017)

13. I. Vaz-Moreira, V. Figueira, A.R. Lopes, R. Pukall, C. Spröer, P. Schumann, O.C. Nunes, C.M. Manaia, Int. J. Syst. Evol. Microbiol. 60, 2415-2419 (2010)

14. A. Marchese, R. Barbieri, M. Pesce, S. Franchelli, A. De Maria, Clin. Microbiol. Infect. 22, 8, 743-744 (2016)

15. J.A. Sáez-Nieto, M.J. Medina-Pascual, G. Carrasco, N. Garrido, M.A. FernandezTorres, P. Villalón, S. Valdezate, New Microbes New Infect. 19, 19-27 (2017)

16. K. Fiedoruk, T. Daniluk, I. Swiecicka, E. Murawska, M. Sciepuk, K. Leszczynska, Genome Announc. 2, 1, 1-2 (2014)

17. T. Ooka, Emerg. Infect. Dis. 18, 3, 488-492 (2012)

18. K.L. Perez, M.J. Alam, A. Castillo, T.M. Taylor, J. Food Prot. 76, 1, 124-128 (2013)

19. D.J. Ingle, O. Clermont, D. Skurnik, E. Denamur, S.T. Walk, D.M. Gordon, Appl. Environ. Microbiol. 77, 8, 2695-2700 (2011)

20. Grabińska-Łoniewska, G. Wardzyńska, E. Pajor, D. Korsak, K. Boryń, Pol. J. Microbiol. 56, 2, 129-138 (2007)

21. J. El-Chakhtoura, E. Prest, P. Saikaly, M. van Loosdrecht, F. Hammes, H. Vrouwenvelder, Water Res. 74, 180-190 (2015)

22. E.P. Holinger, K.A. Ross, Ch.E. Robertson, M.J. Stevens, J.K. Harris, N.R. Pace, Water Res. 49, 225-235 (2014)

23. B. Tan, C. Ng, J.P. Nshimyimana, L.L. Loh, K.Y. Gin, J.R. Thompson, Front. Microbiol. 6, 1-20 (2015)

24. E.I. Prest, J. El-Chakhtoura, F. Hammes, P.E. Saikaly, M.C.M. van Loosdrecht, J.S. Vrouwenvelder, Water Res. 63, 179-189 (2014)

25. D. van der Kooij, H.R. Veenendaal, W.J.H. Scheffer, Water Res. 39, 2789-2798 (2005)

26. A. Rakić, J. Perić, L. Foglar, Ann. Agric. Environ. Med. 19, 3, 431-436 (2012)

27. C.R. Proctor, M. Reimann, B. Vriens, F. Hammes, Water Res. 131, 274-286 (2018) 